\title{
La transparencia publicitaria en los influencers de las redes sociales
}

\section{Advertising transparency in the social media influencers}

\begin{abstract}
Mg. Nataly Guiñez-Cabrera es profesora asistente e investigadora de la Universidad del Bío-Bío (Chile) (nguinez@ubiobio.cl) (https://orcid.org/0000-0002-6109-8457)
\end{abstract}

Lic. Katherine Mansilla-Obando es estudiante del Doctorado en Administración de la Universidad Adolfo Ibáñez (Chile) (kmansilla@alumnos.uai.cl) (https://orcid.org/0000-0003-4273-4259)

Mg. Fabiola Jeldes-Delgado es profesora adjunta e investigadora de la Universidad de Valparaíso (Chile) (fabiola.jeldes@uv.cl) (https://orcid.org/0000-0003-2219-7337)

\begin{abstract}
Resumen
Las redes sociales han contribuido a una nueva estrategia de marketing donde las marcas forjan alianzas con los influencers de las redes sociales para generar contenido publicitario. Esta estrategia conocida como marketing de influencer se encuentra en crecimiento y ganando un interés sustancial en la literatura. Sin embargo, la comprensión actual de la percepción de los influencers de las redes sociales frente a la transparencia del contenido que publicitan sigue siendo limitada, donde prácticamente todos los estudios se centran en la percepción del seguidor y en países desarrollados donde existe mayor regulación frente a la transparencia publicitaria. Para abordar esta problemática, la presente investigación utilizó la teoría de credibilidad de la fuente y la teoría institucional con el objetivo de explorar las percepciones de los influencers de las redes sociales frente a la transparencia del contenido que publicitan. Sobre la base de un estudio cualitativo que incorporó entrevistas semiestructuradas con ocho influencers de las redes sociales en Chile. Los resultados de este estudio muestran que los influencers de las redes sociales consideran relevante en su credibilidad de la transparencia publicitaria; la confiabilidad, la experiencia, la auto-presentación en línea y los aspectos normativos. Estos hallazgos contribuyen a la literatura de marketing de influencer y también tiene importantes implicancias prácticas para el amplio y creciente campo de la publicidad como son los profesionales de marketing, las marcas y los agentes reguladores encargados de proteger al consumidor.
\end{abstract}

\begin{abstract}
Social media has contributed to a new marketing strategy where brands forge alliances with social media influencers to generate advertising content. This strategy is known as influencer marketing, finding itself growing and gaining substantial interest in the literature. However, the current understanding of the perception of social media influencers regarding the transparency of the content they advertise is still limited, where practically all studies focus on the perception of the follower and in developed countries where there is more regulation regarding advertising transparency. To address this issue, this research used the source credibility theory and the institutional theory with the aim of exploring the perceptions of social media influencers regarding the transparency of the content they advertise. Based on a qualitative study that incorporated semi-structured interviews with eight social media influencers from Chile. The results of this study show that social media influencers consider reliability, experience, online self-presentation and the normative aspects relevant in their credibility of advertising transparency. These findings contribute to the influencer marketing literature and have important practical implications for the broad and growing field of advertising, such as marketing professionals, brands and regulators responsible for protecting the consumer.
\end{abstract}

\section{Palabras clave I keywords}

Auto-presentación, confianza, credibilidad, experiencia, influencer, publicidad, seguidor, transparencia. Self-presentation, trust, credibility, experience, influencer, advertising, follower, transparency.

Cómo citar: Guiñez-Cabrera, N., Mansilla-Obando, K., y Jeldes-Delgado, F. (2020). La transparencia publicitaria en los influencers de las redes sociales. Retos Revista de Ciencias de la Administración y Economía, 10(20), pp. 265-281. https://doi.org/10.17163/ret.n20.2020.05 


\section{Introducción}

Se cree que la influencia que tienen los influencers de las redes sociales es bastante poderosa y es probable que siga creciendo en los próximos años (Forbes, 2019). Hasta ahora, la literatura da cuenta que esta influencia se va construyendo en la medida que el influencer cautiva a sus seguidores, a través, de la generación de contenido en las redes sociales donde comparte su personalidad y experiencias (CNBC, 2017; Lou \& Yuan, 2019). Gracias a esta influencia, el influencer cumple un rol vital al atraer a las marcas o empresas y a los profesionales de publicidad (Lou \& Yuan, 2019). Sin embargo, aunque se sabe que los influencers de las redes sociales comparten experiencias relacionadas con marcas de productos, la literatura no ha profundizado sobre la tensión que surge en el influencer de las redes sociales por ser transparente (sobre los beneficios personales por publicitar una marca) y su credibilidad ante los seguidores. Ignorar la percepción de los influencers de las redes sociales frente a transparencia publicitaria impide una mayor comprensión de la credibilidad de esta nueva estrategia de marketing.

Para abordar esta brecha de la literatura, presentamos uno de los primeros estudios en Latinoamérica en proporcionar información desde la perspectiva de los influencers de las redes sociales. Consecuentemente, este estudio intentará responder la siguiente pregunta de investigación: ¿Cuál es la percepción de los influencers de las redes sociales frente a la transparencia del contenido que publicitan? Este estudio espera conocer las dimensiones que los influencers de las redes sociales valoran a la hora de decidir transparentar (o no) la publicidad de su contenido en las redes sociales ante sus seguidores.

Para responder esta pregunta de investigación utilizamos una metodología cualitativa de ocho entrevistas semiestructuradas a influencers de la red social «Instagram», realizadas en el mes de mayo 2019.

Nos basamos en la teoría de «credibilidad de la fuente» (Hovland et al., 1953) que sostiene la confianza, la experiencia, el atractivo y la presentación en línea (redes sociales) del influencer, afectan en su credibilidad ante los seguidores (Djafarova \& Trofimenko, 2018; Ohanian, 1990) y la teoría institucional (DiMaggio \& Powell, 1983). Esperamos que los resultados de este estudio sean acordes con las investigaciones que destacan, por ejemplo, que la confianza y la experiencia del influencer de las redes sociales intervienen positivamente en la percepción de los seguidores frente al patrocinio de un producto o marca (por ejemplo, Amos et al., 2008; Friedman et al., 1976). Asimismo, si consideramos que la conducta de los influencers de las redes sociales afecta las creencias, actitudes y comportamientos de sus consumidores (Dholakia \& Sternthal, 1977; Hovland et al., 1953; Hovland \& Weiss, 1951; Ohanian, 1990), esperamos que sean dimensiones que los influencers valoren a la hora de transparentar la publicidad en su contenido.

Este estudio presenta tres contribuciones. En primer lugar, aportamos a la literatura de marketing de influencer. Al considerar que la literatura anterior se ha enfocado en la perspectiva de los seguidores, por ejemplo, la credibilidad del influencer, desde la óptica del seguidor (Djafarova \& Trofimenko 2018), nuestro estudio ofrece una mirada desde la perspectiva del propio influencer. En segundo lugar, ampliamos la investigación previa al proporcionar evidencia pionera sobre las dimensiones que podrían afectar la 
credibilidad considerando la transparencia publicitaria. Adicionalmente, nos enfocamos en la percepción del influencer de las redes sociales sobre la transparencia publicitaria en un contexto latinoamericano. Finalmente, esta investigación también tiene importantes implicancias prácticas para el amplio y creciente campo de la publicidad, como los profesionales de marketing, las marcas y los entes reguladores encargados de formular políticas públicas de protección al consumidor.

\subsection{Influencers y transparencia publicitaria}

El panorama actual sobre los canales de comunicación masiva y tradicional, como; la televisión, la radio y los medios impresos, ya no son las fuentes de información dominantes para los consumidores (Lou \& Yuan, 2019). Esto ha llevado que los consumidores ya no prestan atención a la publicidad tradicional (Carter, 2016), y utilizan cada vez más las redes sociales para recopilar información sobre la cual basan sus decisiones (Casaló et al., 2018). Así, las empresas reconocen en las redes sociales un recurso estratégico para publicitar sus marcas y construir relaciones sólidas con los usuarios (Shiau, et al., 2018).

A medida que los medios digitales y sociales continúan creciendo en términos de uso, influencia y gastos de publicidad, la industria publicitaria continúa explorando y desarrollando estrategias innovadoras para llegar a sus mercados objetivos (Childers et al., 2018). Una de estas estrategias innovadoras es el marketing de influencer.

\subsubsection{Marketing de influencer}

La administración de las redes sociales es cada vez más relevante en el éxito empresarial (Cano, 2017), y junto con las aplicaciones móviles constituyen un aliado clave para la gestión empresarial en la comunicación y marketing (Aucay \& Herrera, 2017). Siendo posible crear comunidades virtuales de marcas y permitiendo a los usuarios informarse e interactuar con el contenido realizado por la misma (Gutiérrez et al., 2018).

Así, nace el marketing de influencer como una importante estrategia de marketing relativamente nueva, en la que las marcas colaboran o forjan alianzas con personas influyentes de los medios sociales para promocionar sus productos y marcas (De Veirman et al., 2017; Evans et al., 2017).

Un influencer de las redes sociales:

Es ante todo un generador de contenido: alguien que tiene un estado de experiencia en un área específica, que ha cultivado un número considerable de seguidores cautivos, que son de valor de marketing para las marcas, al producir regularmente contenido valioso, a través, de las redes sociales. (Lou \& Yuan, 2019, p. 59)

Los anunciantes le entregan en cierta manera el control al influencer de las redes sociales en la creación de contenido, lo que permite obtener un mensaje que es considerado más auténtico que cuando lo presenta directamente la marca (Childers et al., 2018). A su vez, los consumidores utilizan cada vez más las redes sociales para obtener información sobre productos (Stubb et al., 2019) y guiar sus decisiones de compra, valorando y confiando en las opiniones de sus pares en internet (De Veirman \& Hudders, 2019). Así, los influencers de las redes sociales transmiten su opinión sobre las marcas en sus publicaciones, teniendo el potencial de intervenir en el comportamiento de la audiencia (Stubb \& Colliander, 2019). 
Sin embargo, los influencers de las redes sociales pueden permanecer imprecisos sobre su relación comercial con la marca patrocinadora (De Veirman \& Hudders, 2019). Por lo tanto, el marketing de influencer puede clasificarse como una forma de publicidad nativa, por la naturaleza de la relación pagada entre la marca y el influencer de las redes sociales, que puede no ser del todo clara para la audiencia (Evans et al., 2017). La publicidad nativa es definida como «cualquier publicidad pagada que toma la forma específica y la apariencia del contenido editorial del propio editor» (Wojdynski \& Evans, 2016). El problema con la publicidad nativa es que las personas tienen dificultades para reconocer el intento persuasivo que hay detrás del mensaje (Wojdynski \& Evans, 2016).

Por lo tanto, a medida que el marketing de influencer ha evolucionado hacia una táctica de marketing creciente, el problema con estas publicaciones comerciales en las redes sociales es que se parecen y se mezclan con publicaciones no comerciales y, por lo tanto, los seguidores a menudo no lo reconocen como publicidad (Boerman et al., 2017; Evans et al., 2017).

Así los influencers de las redes sociales deben ser transparentes en cualquier relación comercial (Boerman, 2020). Es necesario incluir etiquetas o transparencias que transmitan efectivamente la naturaleza del mensaje al consumidor para que existan consumidores informados, especialmente cuando la naturaleza pagada del mensaje se ofusca (Evans et al., 2017). Se ha demostrado que las transparencias que son más explícitas tienen más probabilidades de aumentar el reconocimiento de los anuncios (Boerman, 2020; Boerman et al., 2015; Wojdynski \& Evans, 2016). No obstante, aunque se reconoce la relevancia de la transparencia en la relación entre el influencer de las redes sociales y sus seguidores, la literatura del marketing de influencer, se ha centrado en la visión de los seguidores. Específicamente, referente a la percepción de la audiencia sobre la transparencia del patrocinio en las publicaciones de los influencers de redes sociales y el reconocimiento de los anuncios en las respuestas hacia la marca, como las actitudes de la marca y las intenciones de compra por parte de los seguidores (por ejemplo, Boerman, 2020; De Jans et al., 2018; De Veirman \& Hudders, 2019; Evans et al., 2017).

\subsection{Credibilidad de la fuente}

La credibilidad se define como «la medida en que se percibe que la fuente posee experiencia relevante para el tema de la comunicación y se puede confiar en ella para dar una opinión objetiva sobre el tema» (Goldsmith et al., 2000, p. 43). El modelo de "credibilidad de la fuente» sugiere que un mensaje promocional de una fuente creíble, en este caso de un influencer de las redes sociales, afectaría las creencias, actitudes y comportamientos de los consumidores (Dholakia \& Sternthal, 1977; Hovland et al., 1953; Hovland \& Weiss, 1951; Ohanian, 1990). Esta teoría permite explicar o predecir la eficiencia del mensaje (Hovland et al., 1953).

Estudios previos analizan la credibilidad de la fuente en el contexto de los influencers de las redes sociales (por ejemplo, Breves et al., 2019; De Jans et al., 2020; De Veirman \& Hudders, 2019; Stubb \& Colliander, 2019; Stubb et al., 2019; Wojdynski \& Evans, 2016).

Si bien, el modelo de «credibilidad de la fuente» sugiere que la eficiencia del mensaje comunicado por un patrocinador depende de la percepción de los consumi- 
dores sobre la experiencia, la confiabilidad y el atractivo del patrocinador del producto o marca (Amos et al., 2008; Friedman et al., 1976; Hovland \& Weiss, 1951; Ohanian, 1990), el estudio previo de Newell y Goldsmith (2001) considera la confiabilidad y experiencia como dimensiones propias del modelo de credibilidad de la fuente. A continuación, se presenta una descripción general de las dimensiones del constructo «credibilidad de la fuente» a utilizar.

\subsubsection{Confiabilidad}

La «confiabilidad percibida» se define como los atributos de dignidad y honestidad que posee una fuente (Friedman et al., 1976). La confianza en la comunicación es el grado de confianza del oyente y el nivel de aceptación del hablante y del mensaje (Ohanian, 1990). Hovland et al. (1953) denominaron «fuente de credibilidad» a la confianza de un oyente en un hablante. Cuando un consumidor cree que una fuente es confiable, también supone que el mensaje comunicado es altamente creíble (Hovland \& Weiss, 1951). Ohanian (1990) indicó que la confiabilidad del comunicador (por ejemplo, un influencer de las redes sociales) es una construcción importante en la investigación de persuasión y cambio de actitud.

\subsubsection{Experiencia}

Es la segunda dimensión de la «credibilidad de la fuente» que definió (Hovland et al., 1953). Las investigaciones sobre la experiencia de la fuente en la comunicación persuasiva indican que la experiencia percibida tiene un efecto positivo en el cambio de actitud (Ohanian, 1990). El nivel de experiencia también depende de las percepciones de la audiencia (Hovland et al., 1953; Ohanian, 1990). Si se percibe como una fuente con valiosa información y no necesariamente como un experto en el producto vendido, el mensaje será más persuasivo y generará reacciones más favorables hacia el producto y mayores intenciones de compra (Erdogan, 1999; Ohanian, 1990). En el estudio de influencers de redes sociales de Djafarova y Trofimenko (2018), los entrevistados se refirieron a «competencia» en lugar de «experiencia».

\subsubsection{Auto-presentación en línea}

Esta dimensión fue incorporada en el estudio de Djafarova y Trofimenko (2018). La «auto-presentación» proviene de la psicología social y es la forma en que una persona quiere ser percibida por los demás, existiendo una presión para auto-presentarse como un yo mejorado o deseado, según lo que espera la audiencia, y así parecer más creíble (Goffman, 1959).

Las redes sociales han generado un interés en la auto-presentación (DeAndrea \& Walther, 2011), ya que ésta ayuda a obtener aceptación por parte de los otros (Chiang \& Suen, 2015). Sin embargo, cuando las personas perciben las auto-presentaciones como engañosas se producen actitudes negativas (Schlenker \& Weigold, 1992). Por lo anterior existe una necesidad de establecer la credibilidad mediante la auto-presentación (Ellison et al., 2006). En el caso de los influencers de las redes sociales, la auto-presentación que tengan en redes sociales influirá en la credibilidad percibida por parte de la audiencia (Djafarova \& Trofimenko, 2018). 


\subsection{Teoría institucional}

Esta perspectiva teórica señala que el entorno institucional está compuesto por instituciones que ejercen presión a las estructuras y prácticas de determinados actores sociales (DiMaggio \& Powell, 1983). Los actores sociales pueden ser empresas, consumidores, asociados comerciales, gobiernos; quienes dan forma a las normas institucionales y, al mismo tiempo, dan una respuesta a ellas. Por consiguiente, los actores sociales, en este caso, los influencers de las redes sociales, se ajustan y buscan cumplir las presiones institucionales para lograr y aumentar su legitimidad, y su capacidad de supervivencia (DiMaggio \& Powell, 1983).

Esta teoría propone la presión normativa (DiMaggio \& Powell, 1983). Las presiones normativas se refieren a la estructura formal o informal del actor social, en este caso el influencer de las redes sociales ante el cumplimiento de una norma (Nwabueze \& Mileski, 2008), específicamente cómo se deberían hacer las cosas de forma legítima al perseguir determinados valores (Scott, 1995).

\section{Materiales y método}

Siendo los influencers de las redes sociales un fenómeno muy poco explorado, el diseño de este estudio, adoptó un enfoque cualitativo, que es un método adecuado que proporciona mucha información y comprensión de fenómenos poco investigados (Corbin \& Strauss, 2015). Así podremos dar respuesta a nuestra pregunta de investigación para conocer la percepción de los influencers de las redes sociales con respecto al transparencia publicitaria de su contenido en las redes sociales, específicamente en la red social Instagram.

Con el fin de recopilar la fuente de datos primaria, se realizaron entrevistas semiestructuradas. Una ventaja de usar este tipo de entrevistas es la flexibilidad que se le brinda al entrevistador, pudiendo agregar preguntas que se consideran relevantes a lo largo de la entrevista o profundizar en cualquier punto que se considere esencial para responder la pregunta de investigación y, además, la información se recopila desde la perspectiva del entrevistado, lo cual es muy enriquecedor y proporciona información más detallada (Corbin \& Strauss, 2015; Creswell, 2013).

Se aplicó una muestra de bola de nieve (Creswell, 2013), donde el primer entrevistado nos dio información para contactar a los siguientes participantes del estudio que eran influencers activos de la red social Instagram. Se utilizó la plataforma de Instagram, porque actualmente es la más utilizada en marketing de influencer (WFA, 2018) y Chile fue el país seleccionado como un contexto interesante para realizar las entrevistas, ya que $87,5 \%$ de los hogares tienen acceso a Internet, que es un porcentaje mucho más alto que el de otros países de Latinoamérica (OECD, 2019).

Los participantes que fueron recomendados por el primer entrevistado, fueron contactados por la misma red social Instagram vía mensaje de texto, ya que es una forma fácil y directa de poder comunicarse. En el mensaje se explicó el objetivo de la investigación y los procedimientos de la entrevista, finalmente 8 influencers de las redes sociales acordaron participar en el estudio de un total de 19 que se contactaron. Los influencers de las redes sociales fueron entrevistados independientemente de los productos que publicitan. Las entrevistas se llevaron a cabo en el mes de mayo de 2019. Los detalles de los participantes se presentan en la tabla 1. 
Antes de la recolección de datos, se realizaron dos entrevistas de prueba para verificar la estructura y la comprensión de las preguntas. Estas entrevistas de prueba fueron realizadas a dos influencers de las redes sociales, que no fueron considerados dentro de la muestra final. Luego, el instrumento fue modificado con las observaciones identificadas y finalmente, la entrevista fue revisada y perfeccionada por un experto en investigación cualitativa.

La recopilación de datos se realizó por medio de entrevistas semiestructuradas cara a cara (presencial) y por vía telefónica, que se detalla en la tabla 1. Las entrevistas se realizaron presencialmente cuando los participantes estaban dispuestos a reunirse, se llevaron a cabo en un entorno elegido por los entrevistados, que en su mayoría era en su lugar de trabajo, por la conveniencia de los participantes. Se realizaron telefónicamente a aquellos influencers de las redes sociales que no podían realizarla presencialmente por su gran agenda de compromisos y poca disponibilidad de tiempo. Las entrevistas fueron realizadas por uno de los miembros del equipo de investigación, quién también formó parte del diseño de la guía de la entrevista.

Las entrevistas se realizaron en el idioma español. Al comienzo de las entrevistas, se les informo a cada entrevistado de la confidencialidad de su identidad y la autorización de la grabación de audio de la entrevista. Los nombres de los participantes fueron modificados en este estudio, para mantener la confidencialidad de los datos personales. Las entrevistas duraron un promedio de 15 minutos, fueron grabadas en audio y transcritas textualmente por los tres investigadores para facilitar el análisis de los datos (Miles et al., 2019).

La estructura de la entrevista contenía una serie de preguntas abiertas estandarizadas para guiar el proceso. Inicialmente, se presentó una breve introducción del tema a tratar y una pregunta general de calentamiento sobre la relación que el entrevistado tiene con las redes sociales. Luego, se hicieron preguntas preliminares sobre los antecedentes personales del participante. Posteriormente, se le pidió al entrevistado que indicara si realiza transparencia de la publicidad en el contenido que crea (si utiliza «hashtag \#») y comparte en su cuenta de Instagram. Esta pregunta fue diseñada para identificar por qué razón transparentan o no la publicidad en las redes sociales (Miles et al., 2019). Después de identificar algunos factores que respondieron a la pregunta de investigación, se les pidió a los participantes que profundizaran su respuesta. En algunas ocasiones, el participante se desvió hacia otras respuestas, y el entrevistador lo dirigió nuevamente al objetivo principal del estudio, que es lograr que el participante responda libremente sobre su experiencia con la transparencia publicitaria.

Las entrevistas alcanzaron su punto de saturación en la entrevista número ocho, sin obtener información adicional, lo que permitió completar el proceso de recolección de datos en este punto (Silverman, 2000). Para la interpretación y el análisis de los datos, se llevó a cabo lo sugerido por Spiggle (1994) y Corbin y Strauss (2015). Primero los investigadores se sumergieron en los datos, leyendo cuidadosamente cada una de las transcripciones. Posteriormente, se realizó un desglose manual con las frases claves resaltadas que codifican las unidades primarias. Luego, se realizó la categorización, clasificando cada grupo de datos relacionados con los motivos de transparentar la publicidad en el contenido. Posteriormente, en una etapa superior de abstracción, se redujo el número de categorías, agrupando aquellas que tenían 
similitudes. A continuación, se efectuó una comparación, y se decidió qué categorías eran las más relevantes. Subsiguientemente, para triangular los datos, se revisaron fuentes secundarias como; artículos relevantes de prensa y la revisión del contenido de los perfiles de Instagram de los influencers de las redes sociales. Finalmente, en la etapa de integración, los investigadores dieron sentido a los datos en las inferencias, estos se usaron para generar una conexión con las preguntas de investigación y con el marco teórico presentado en la sección anterior (Spiggle, 1994). Los hallazgos de esta investigación se describen a continuación.

Tabla 1. Caracterización de los influencers de las redes sociales entrevistados

\begin{tabular}{|c|c|c|c|c|c|c|c|}
\hline & Género & "ृ & 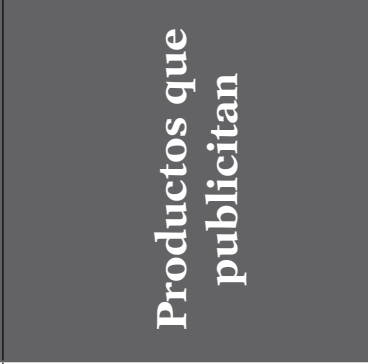 & 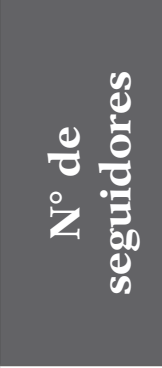 & $\begin{array}{l}\frac{5}{0} \\
\frac{0}{0} \\
\frac{0}{0} \\
6 \\
0 \\
0\end{array}$ & 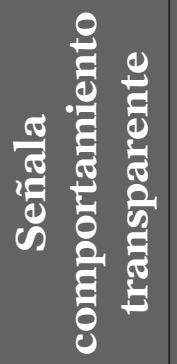 & 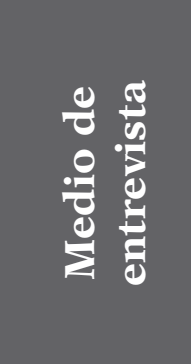 \\
\hline A & Femenino & $\begin{array}{c}27 \\
\text { años }\end{array}$ & $\begin{array}{l}\text { Belleza, moda, } \\
\text { viajes y } \\
\text { alimentación. }\end{array}$ & 170000 & $\begin{array}{l}\text { Modelo y } \\
\text { comunicadora } \\
\text { feminista }\end{array}$ & Sí & Presencial \\
\hline B & Masculino & $\begin{array}{c}30 \\
\text { años }\end{array}$ & $\begin{array}{l}\text { Música, moda, } \\
\text { alimentación. }\end{array}$ & 129000 & Músico & No & Telefónico \\
\hline $\mathrm{C}$ & Masculino & $\begin{array}{c}26 \\
\text { años }\end{array}$ & $\begin{array}{l}\text { Belleza, } \\
\text { alimentación y } \\
\text { deporte. }\end{array}$ & 10500 & Modelo & No & Telefónico \\
\hline D & Femenino & $\begin{array}{c}28 \\
\text { años }\end{array}$ & $\begin{array}{l}\text { Belleza, moda, } \\
\text { viajes, alimentación } \\
\text { y deporte. }\end{array}$ & 197000 & Emprendedora & No & Presencial \\
\hline $\mathrm{E}$ & Femenino & $\begin{array}{c}30 \\
\text { años }\end{array}$ & $\begin{array}{l}\text { Belleza, } \\
\text { moda, viajes y } \\
\text { alimentación. }\end{array}$ & 16000 & $\begin{array}{l}\text { Modelo, } \\
\text { médico. }\end{array}$ & No & Presencial \\
\hline $\mathrm{F}$ & Femenino & $\begin{array}{c}34 \\
\text { años }\end{array}$ & $\begin{array}{l}\text { Belleza, } \\
\text { moda, viajes y } \\
\text { alimentación. }\end{array}$ & 976000 & Modelo, actriz. & Sí & Telefónico \\
\hline G & Femenino & $\begin{array}{c}37 \\
\text { años }\end{array}$ & $\begin{array}{l}\text { Alimentación y } \\
\text { deporte. }\end{array}$ & 11700 & Chef & Sí & Presencial \\
\hline $\mathrm{H}$ & Femenino & $\begin{array}{c}34 \\
\text { años }\end{array}$ & $\begin{array}{l}\text { Belleza, moda y } \\
\text { viajes. }\end{array}$ & 30300 & $\begin{array}{l}\text { Diseñadora } \\
\text { web }\end{array}$ & No & Presencial \\
\hline
\end{tabular}

Fuente: Elaboración propia. Entrevistas realizadas en mayo 2019.

\section{Resultados}

Los datos revelan que los influencers de las redes sociales entrevistados consideran la transparencia publicitaria relevante para su actividad. Primero, los datos identifican las dimensiones del modelo de credibilidad de la fuente en línea: confiabilidad, experiencia (Amos et al, 2008; Friedman et al., 1976; Hovland \& Weiss, 
1951; Ohanian, 1990) y la auto-presentación en línea (Djafarova \& Trofimenko, 2018), dimensiones que se encontraron en estudios previos de credibilidad de los influencers de las redes sociales (por ejemplo, Breves et al. 2019; De Jans et al., 2020; De Veirman \& Hudders, 2019; Stubb \& Colliander, 2019; Stubb et al., 2019; Wojdynski \& Evans, 2016). Segundo, los datos identifican un aspecto específico de la transparencia publicitaria de los influencers de las redes sociales que corresponde al carácter normativo.

\subsection{Dimensiones del modelo de credibilidad de la fuente}

\subsubsection{Confiabilidad}

La confianza en la comunicación es el grado de confianza del oyente y el nivel de aceptación del hablante y del mensaje (Ohanian, 1990). Según Breves et al. (2019), un influencer de las redes sociales debe ser cauteloso y debe incorporar altos niveles de autenticidad en sus publicaciones, incluso se debe mostrar congruencia entre el influencer de las redes sociales y la marca que publicita. En este estudio, el influencer de las redes sociales percibe que los seguidores confían en el contenido que ellos publican, principalmente porque los asocian a las marcas que publicitan. Por ejemplo, el entrevistado E indica:

Yo nunca planeé ser influencer y creo que se dio porque la gente confía en lo que, recomiendo, por lo mismo trabajo con marcas que realmente me gustan y me representan. (Entrevistado E)

En específico, la confiabilidad se refiere a la honestidad, integridad y credibilidad del endosante (Erdogan, 1999), como también a la moralidad de una fuente en el suministro de información objetiva e imparcial (Flanagin \& Metzger, 2007). Si bien, existen seguidores como los adolescentes que no son conscientes de que la publicación en las redes sociales sea publicidad (De Jans et al., 2020), existen estudios que señalan que un influencer al que se le paga para recomendar una marca en las redes sociales puede ser menos propenso a expresar una opinión honesta sobre el producto y, por lo tanto, sea parcial. Como resultado, los consumidores pueden sentirse engañados (Campbell \& Kirmani, 2000). A pesar de ello, cuando las recomendaciones de productos surgen desde personas influyentes, éstas son percibidas como auténticas y honestas (De Veirman et al., 2017). En este estudio, los influencers de las redes sociales perciben que son honestos con la información que transmiten a sus seguidores. Siendo actores intermediarios de una marca que publicitan y respaldan mediante el «hashtag \#» en sus contenidos. Por ejemplo, el entrevistado B declara:

La gente también agradece saber que le estamos publicitando algo, cachaí, no como que estamos intentando mentirles, y la gente ya se da cuenta que uno está haciendo publicidad de algo, entonces no sé, eh... ventajas, que nosotros como influencers estamos siendo 100\% honestos con las personas que están viendo aquel producto. (Entrevistado B)

Incluso, un influencer de las redes sociales señala que al utilizar el «hashtag \#» se está siendo informativo y auténtico a la vez, al no engañar a sus seguidores. Como indica:

Al final, uno no está engañando a nadie, es obvio que es una publicidad y que a uno le están pagando por eso. Aparte hay un montón de contenido que uno hace eh... porque quiere simplemente mostrar faceta. (Entrevistado F) 
Es más, evidencia empírica previa señala que la justificación del patrocinio genera más actitudes positivas desde los consumidores hacia las personas influyentes, aumentando la credibilidad de la fuente y el mensaje (Stubb et al., 2019). Si bien, Wojdynski et al. (2018) se refieren a la transparencia del patrocinio como la medida en que una comunicación patrocinada notifica al espectador sobre la naturaleza de pago y la identidad del patrocinador. Se conoce, al menos en los blogs, que los lectores aprecian la transparencia y a los blogueros que aceptan patrocinio siendo percibidos como altamente creíbles, pero si éstos no brindan información sobre patrocinio o utilizan expresiones vagas puede generar sospechas entre los lectores y reducir así la credibilidad de la fuente (Carr \& Hayes, 2014). Los resultados señalan que los influencers de las redes sociales no están de acuerdo con transparentar sus contenidos. Uno de los participantes señala que, aunque recibe remuneración por publicitar productos/servicios, a través, de la red social Instagram, esto no significa que deba indicar que es publicidad. Para el influencer de las redes sociales, los productos que publicita son productos que utiliza con frecuencia y siempre han sido de su agrado. Participante D señala:

No me gusta mucho porque eh... yo sí trabajo con marcas, a mí sí me pagan marcas por hacerles publicidad, pero son marcas que yo he usado toda la vida, que, aunque me paguen o no, yo igual voy las voy a mostrar usándolas, es como... tu toda la vida te has maquillado con Mac o toda la vida has tomado Coca-Cola... y un día llega Coca-Cola y te dice... sigue tomando Coca-Cola, pero aparte te quiero pagar para que uses mi marca, ¿qué vas a decir tú? (Entrevistado D)

\subsubsection{Experiencia}

Ohanian (1990) señala que la experiencia se asocia a un cambio positivo en la actitud. La experiencia se refiere al conocimiento, la experiencia y a las habilidades del endosante (Erdogan, 1999). Las habilidades que los influencers demuestran en las redes sociales pueden representar una fuente persuasiva y favorable para la intención de compra del consumidor (Djafarova \& Trofimenko, 2018). Además, estudios previos señalan que las recomendaciones de productos publicadas por personas influyentes se perciben como sinceras si se perciben como expertos en el campo (De Veirman et al., 2017).

Por lo tanto, al analizar los datos hemos percibido dos tipos de experiencias. Primero, aquella relacionada a la experiencia profesional previa al convertirse en influencer de las redes sociales y segundo, a la experiencia del influencers de las redes sociales con productos publicitarios. Respecto a la primera, se identifican algunos influencers de las redes sociales que perciben que su experiencia previa en el contexto laboral de comunicaciones les ha permitido ver la transparencia de la información que entregan de manera más responsable y, por lo tanto, esto ha llevado a generar cierto respeto en los seguidores y validez frente a la información que ellos entregan en sus redes. Por ejemplo, el influencer de redes sociales F declara:

Yo siento que para las personas profesionales que vienen de la televisión, radio, del rubro de las comunicaciones, esto no es nuevo, es algo que lo hacemos día a día. (Entrevistado F)

Respecto a la segunda, los influencers de las redes sociales indican que ellos reflejan en sus contenidos publicitarios las experiencias que han tenido con productos 
y servicios, y cómo el rol del «hashtag \#» permite su actuar en búsqueda de la autenticidad. Como el influencer D que señala que solo publicita marcas que utiliza.

Entonces por eso yo no estoy de acuerdo, porque yo por lo menos dentro de mis filtros es promocionar marcas que realmente use y que realmente me gusten. (Entrevistado D)

\subsubsection{Auto-presentación en línea}

Djafarova y Trofimenko (2018) indica que la auto-presentación en línea que tenga el influencer de las redes sociales influirá en la credibilidad percibida por parte de la audiencia. Algunos influencers de las redes sociales entrevistados señalan que, al estar en las redes sociales, los influencers pueden presentarse de una forma $\mathrm{u}$ otra, es decir, pueden experimentar cambios de rol. Como señala el influencer de las redes sociales E, según el contenido que generan y la transparencia percibida por sus seguidores pueden ser percibidos como un ente de opinión o como un vendedor.

He visto muchos influencer que al final uno entra a sus Instagram y son pura publicidad. Bueno, bien por ellos, pero parecen vendedores en vez de influencers y creo que es abuso de su poder como rostro. Ventaja, trasparentar que uno está trabajando con una marca. Desventaja, pasar a ser vendedor. (Entrevistado E)

Así, hay influencers que exigen en su labor y asociación con las marcas ser auténticos y mostrarse en sus contenidos de las redes sociales tal cual ellos son. Como indica el entrevistado $\mathrm{H}$, que exige demostrar un comportamiento según su escala de valores, para así demostrar legitimidad ante sus seguidores.

Siempre que tengo algún tipo como de oportunidad de tener una campaña pagada, les explico esto, que yo siempre pongo que es publicidad o que fue regalado el producto, lo que sea en mis stories, en los posts y en el blog. Y van varias campañas que me han cancelado por eso, que me dicen "no, pero quítalo" y yo les digo "no, no lo puedo quitar", "ah sí, no lo quitas entonces te sales de la campaña” y me salgo de la campaña iiijajaja!!! Entonces, por eso es que no tengo tanto trabajo porque yo siempre exijo decir la verdad y mucha gente me dice como que nadie se va a dar cuenta mostrar legitimidad en el trabajo. (Entrevistado H)

Incluso, el mismo influencer de las redes sociales señala que al tener una relación tan cercana con sus seguidores, ha causado ser más comprometido y que ha generado buenas intenciones al comportarse de manera honesta con ella misma y con su trabajo.

Pero tal vez sí ganaría más de ello, pero al final como que me aferro a mis valores y dejo de estar haciendo esto y también les explico a ellos de que no es una, no me importa si mis seguidores saben que es publicidad o no, porque yo tengo una relación súper cercana con ellos. Pero a mí me importa a mí. Como yo saber que estoy siendo honesta conmigo y mi trabajo. (Entrevistado H)

Dado lo anterior, es que los influencers de las redes sociales simplemente prefieren imitar a otros en sus comportamientos, sin interés de por medio, como lo indica el participante G:

Pero me parece que está bien, yo si lo pudiera hacer lo haría también y creo que no afecta para nada la actividad de los influencers, ni a mí ni a nadie. (Entrevistado C) 


\subsection{Extensión a las dimensiones del modelo de credibilidad de la fuente usando la teoría institucional}

La comunicación se asocia al nivel de aceptación del hablante y del mensaje (Ohanian, 1990), es decir, el grado de credibilidad que los influencers de las redes sociales perciben desde el actuar y el comportamiento de los seguidores respecto a la publicidad y contenido entregado. Por lo tanto, la percepción de credibilidad del contenido comunicado por el influencer de las redes sociales en el contexto de transparencia publicitaria, surge de las presiones a las estructuras y prácticas de determinados actores sociales (DiMaggio \& Powell, 1983). Los influencers de las redes sociales perciben presiones normativas respecto a las marcas patrocinadoras e instituciones reguladoras, para generar contenido más transparente.

\subsubsection{Carácter normativo asociado a marcas patrocinadoras}

Los influencers de las redes sociales entrevistados señalan que, al demostrar un comportamiento transparente en sus avisos publicitarios, trae consigo consecuencias con las marcas patrocinadoras. Como señala el influencer de las redes sociales $\mathrm{H}$, por querer ser transparente mediante la honestidad perdió una campaña publicitaria.

Si no lo estoy haciendo por los demás, lo hago por mí, es una cosa de honestidad propia que yo sé que eso es publicidad. Y me sacan de las campañas por eso, de hecho, acabo de perder una la semana pasada. (Entrevistado $\mathrm{H}$ )

\subsubsection{Carácter normativo asociado a instituciones reguladoras}

Un aspecto relevante en el análisis de los datos son las regulaciones bajo las cuales están sujetos los influencers de las redes sociales. El influencer B considera como aspecto positivo que el SERNAC (servicio nacional del consumidor en Chile) pueda obtener información de su labor mientras se rige bajo las reglas establecidas de tal institución. Esta institución y la ley del consumidor permiten regular y promover las buenas prácticas en la transparencia de los mercados en el contexto chileno. Participante B declara:

Entonces la gente del SERNAC puede ver con qué marcas estamos trabajando y que lo que estamos haciendo —en el fondo- está siendo declarado. (Entrevistado B)

Además, el influencer de las redes sociales asocia lo normativo a otros contextos, comparando el uso del «hashtag \#» con otras modalidades de transparencia. Como indica el entrevistado G, que en Estados Unidos los influencers de redes sociales transparentan sus contenidos mediante el: «Patrocinado por».

Me gusta más como funciona por ejemplo en Estados Unidos donde existe el... el... como debajo del nombre de la persona. Dice patrocinador por... (Entrevistado G)

La figura 1 muestra el marco conceptual que propone nuestra investigación, basada en la credibilidad de la fuente en línea de Djafarova y Trofimenko (2018) y la teoría Institucional (DiMaggio \& Powell, 1983). 


\section{Figura 1. Marco conceptual de la percepción de la transparencia publicitaria median- te la credibilidad de los influencers de las redes sociales}
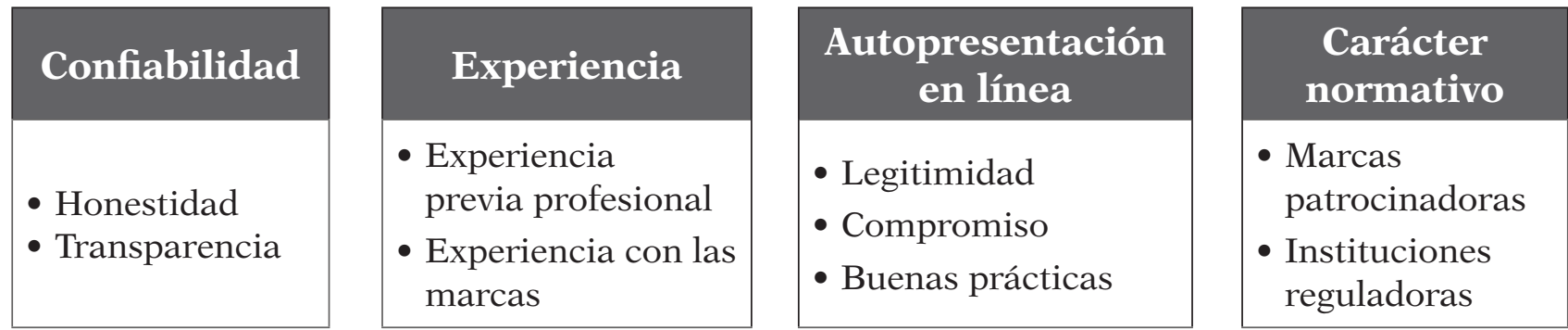

Fuente: Elaboración propia. Basado en las entrevistas realizadas en mayo 2019 y en el modelo de la credibilidad de la fuente en línea de Djafarova y Trofimenko (2018) y la teoría Institucional (DiMaggio \& Powell, 1983).

\section{Discusión y conclusiones}

La contribución de esta investigación fue identificar las percepciones que tienen los influencers de las redes sociales con respecto a la transparencia de la publicidad. Como primer acercamiento, observamos en los resultados de las entrevistas que la percepción de los influencers de las redes sociales sobre la transparencia publicitaria en sus redes no es homogénea. Cerca de un $40 \%$ de los entrevistados percibe que está actuando de forma transparente sobre la publicidad de los productos en sus contenidos, mediante la declaración del «hashtag \#». Contrariamente, más del 30\% percibe que no es necesario transparentar la publicidad y que no hay nada negativo al omitirlo. Esta primera aproximación al conocer la percepción sobre la transparencia publicitaria que tienen los influencers de redes sociales en su contenido, nos permite visualizar que existe mucho por avanzar en estos temas.

En específico, en base a la teoría de la credibilidad de la fuente nuestros resultados identifican tres de sus dimensiones. Primero, se identificó que la dimensión de confiabilidad se vincula a la honestidad que los influencers de las redes sociales rescatan en la relación con sus seguidores. La percepción de los influencers de redes sociales sobre la confiabilidad de la publicidad se asocia a un atributo de honestidad y de transparencia, las cuales son valoradas por sus seguidores (en línea con Wojdynski et al., 2018; Carr \& Hayes, 2014).

Segundo, en la dimensión de experiencia, los influencers de las redes sociales perciben que su experiencia profesional previa y su experiencia en trabajar con marcas, les entrega habilidades persuasivas en la transparencia de la publicidad. En otras palabras, cuando existe conocimiento del contenido que se comparte, los seguidores tenderían a tener una mayor credibilidad en el influencer de las redes sociales. En línea con De Veirman y otros (2017), los influencers de redes sociales creen que para los seguidores es más relevante la valiosa información como experto que pueden entregar sobre un producto, que transparentar que se trata de publicidad.

Tercero, en la dimensión de la auto-presentación en línea, que se relaciona con la imagen que se quiere proyectar a los seguidores teniendo en mente cómo ellos esperan que el influencer de las redes sociales sea. Los participantes de esta investigación declaran que tanto, por resguardar su propio sistema valórico, como lo que esperan los seguidores, no ven beneficioso omitir cuando se trata de publicidad de un produc- 
to en su generación de contenido. Causando un mayor compromiso y el desarrollo de buenas prácticas hacia sus seguidores.

Además, al igual que el estudio de Newell y Goldsmith (2001) nuestra investigación no identifica la dimensión de atractivo en la credibilidad de la fuente en la transparencia publicitaria de los influencers de las redes sociales.

Por último, la extensión a las dimensiones del modelo de la credibilidad de la fuente corresponde al carácter normativo. Asociado a esta dimensión es pertinente mencionar que los influencers de las redes sociales quieren responder a las presiones normativas establecidas sobre la obligatoriedad de transparentar la publicidad. De este modo, los influencers de las redes sociales muestran una imagen de respeto por las normas que, aunque no es transversal para todos los países, los influencers de las redes sociales están en su mayoría de acuerdo con acatarlas. En este caso, los influencers de las redes sociales, perciben que los aspectos normativos se asocian tanto a las marcas patrocinadoras como a las instituciones reguladoras.

Bajo esta identificación de las dimensiones, podemos concluir que confirmamos estudios previos que analizan la transparencia publicitaria con la credibilidad de la fuente de los influencers de las redes sociales (por ejemplo, Breves et al. 2019; De Jans et al., 2020; De Veirman \& Hudders, 2019; Djafarova \& Trofimenko, 2018; Stubb \& Colliander, 2019; Stubb et al., 2019; Wojdynski \& Evans, 2016) confirmando la experiencia, la confiabilidad y la auto-presentación en línea desde la perspectiva del influencer de la redes sociales, extendiendo el modelo con la incorporación del carácter normativo. Estos resultados fueron ilustrados en la Figura 1.

Este estudio enriquece la literatura actual sobre el marketing de influencer, buscando conocer estas perspectivas con respecto a la transparencia publicitaria desde la perspectiva el influencer de las redes sociales. Además, contribuimos al modelo de la credibilidad de la fuente al conectarla con la teoría institucional para analizar la transparencia publicitaria del contenido de los influencers de las redes sociales.

Si bien este enfoque podría ser actual y muy común en los países desarrollados, este artículo representa uno de los primeros estudios en una economía emergente, como lo es Chile.

Nuestra investigación posee limitaciones en la muestra que se encuentra en un solo contexto, por lo que los datos no pueden ser generalizables. Con respecto a las investigaciones futuras, podemos señalar que, si bien este estudio entregó primeros indicios de la credibilidad del influencer de las redes sociales en la transparencia publicitaria, los estudios comparativos podrían agregar ideas adicionales. Por ejemplo, considerar diferentes contextos en Latinoamérica, o analizar el efecto de la transparencia publicitaria en diferentes marcas patrocinadoras.

Finalmente, las implicaciones prácticas para los tomadores de decisiones como los profesionales de marketing, es conocer la percepción desde la mirada del propio influencer de las redes sociales, lo que podría considerar el aspecto normativo, motivando a estos entes de opinión a indicar de manera explícita y transparente la publicidad para mejorar la credibilidad. Así, también en el caso de los agentes reguladores, este estudio permite conocer que varios influencers de las redes sociales se rigen principalmente por el aspecto normativo, por lo tanto, se deberían crear normas o políticas públicas más estrictas para proteger a los consumidores de cualquier tipo de engaño o poca transparencia de la publicidad en las redes sociales. 


\section{Referencias}

Amos, C., Holmes, G., \& Strutton, D. (2008). Exploring the relationship between celebrity endorser effects and advertising effectiveness: A quantitative synthesis of effect size. International Journal of Advertising, 27(2), 209-234. https://doi.org/10.1080/02650487.2008.11073052

Aucay, E., \& Herrera, P. (2017). Nivel de uso de las redes sociales en el proceso de comunicación en las MIPES de Cuenca. Retos, 14(8), 81-98. http://dx.doi.org/10.17163/ret.n14.2017.04

Boerman, S.C. (2020). The effects of the standardized Instagram disclosure for micro- and meso-influencers. Computers in Human Behavior, 103, 199-207. https://doi.org/10.1016/j.chb.2019.09.015

Boerman, S.C., Van Reijmersdal, E.A., \& Neijens, P. C. (2015). Using eye tracking to understand the effects of brand placement disclosure types in television programs. Journal of Advertising, 44(3), 196-207. https://doi.org/10.1080/00913367.2014.967423

Boerman, S.C., Willemsen, L.M., \& Van Der Aa, E.P. (2017). "This post is sponsored": Effects of sponsorship disclosure on persuasion knowledge and electronic word of mouth in the context of Facebook. Journal of Interactive Marketing, 38, 82-92.

https://doi.org/10.1016/j.intmar.2016.12.002

Breves, P.L., Liebers, N., Abt, M., \& Kunze, A. (2019). The perceived fit between instagram influencers and the endorsed brand: How influencer-brand fit affects source credibility and persuasive effectiveness. Journal of Advertising Research, 59(4), 440-454.

https://doi.org/10.2501/JAR-2019-030

Campbell, M. C., \& Kirmani, A. (2000). Consumers' use of persuasion knowledge: The effects of accessibility and cognitive capacity on perceptions of an influence agent. Journal of Consumer Research 27(1), 69-83. https://doi.org/10.1086/314309

Cano, R. (2017). Técnicas de marketing de los clubes de fútbol de élite en las redes sociales. Retos, 13(7), 43-58. https://doi.org/10.17163/ret.n13.2017.03

Carr, C.T., \& Hayes, R.A. (2014). The Effect of Disclosure of Third-Party Influence on an Opinion Leader's Credibility and Electronic Word of Mouth in Two-Step Flow. Journal of Interactive Advertising, 14(1), 38-50. https://doi.org/10.1080/15252019.2014.909296

Carter, D. (2016). Hustle and Brand: The Sociotechnical Shaping of Influence. Social Media + Society, 2, 1-12. https://doi.org/10.1177/2056305116666305

Casaló, L.V., Flavián, C., \& Ibáñez-Sánchez, S. (2018). Influencers on Instagram: Antecedents and consequences of opinion leadership. Journal of Business Research, 117, 510-519. https://doi.org/10.1016/j.jbusres.2018.07.005

Chiang, J.K.H., \& Suen, H.Y. (2015). Self-presentation and hiring recommendations in online communities: Lessons from LinkedIn. Computers in Human Behavior, 48, 516-524. https://doi.org/10.1016/j.chb.2015.02.017

Childers, C.C., Lemon, L.L., \& Hoy, M.G. (2018). \#Sponsored \#Ad: Agency Perspective on Influencer Marketing Campaigns. Journal of Current Issues and Research in Advertising, 40(3), 258-274. https://doi.org/10.1080/10641734.2018.1521113

CNBC (2017). “Social Media Mavens Wield 'Influence', and Rake in Big Dollars” August 12, 2017. https://bit.ly/SMIinfluence

Corbin, J., \& Strauss, A. (2015). Basics of Qualitative Research. Sage Publications.

Creswell, J.W. (2013). Qualitative inquiry and research design: choosing among five approaches. Sage Publications.

DeAndrea, D.C., \& Walther, J.B. (2011). Attributions for inconsistencies between online and offline self-presentations. Communication Research, 38(6), 805-825. https://doi. org/10.1177/0093650210385340

De Jans, S., Cauberghe, V., \& Hudders, L. (2018). How an Advertising Disclosure Alerts Young Adolescents to Sponsored Vlogs: The Moderating Role of a Peer-Based Advertising Literacy Intervention through an Informational Vlog. Journal of Advertising, 47(4), 309-325. https://doi. org/10.1080/00913367.2018.1539363

De Jans, S., Van de Sompel, D., De Veirman, M., \& Hudders, L. (2020). \#Sponsored! How the recognition of sponsoring on Instagram posts affects adolescents' brand evaluations through source 
evaluations. Computers in Human Behavior, 109 https://doi.org/10.1016/j.chb.2020.106342

De Veirman, M., Cauberghe, V., \& Hudders, L. (2017). Marketing through Instagram influencers: The impact of number of followers and product divergence on brand attitude. International Journal of Advertising, 36(5), 798-828. https://doi.org/10.1080/02650487.2017.1348035

De Veirman, M., \& Hudders, L. (2019). Disclosing sponsored Instagram posts: the role of material connection with the brand and message-sidedness when disclosing covert advertising. International Journal of Advertising, 39(1), 94-130. https://doi.org/10.1080/02650487.2019.1575108

Dholakia, R., \& Sternthal, B. (1977). Highly credible source: Persuasive facilitator or persuasive liabilities? Journal of Consumer Research, 3(4), 223-232. https://doi.org/10.1086/208671

DiMaggio, P.J., \& Powell, W.W. (1983). The Iron Cage Revisited: Institutional Isomorphism and Collective Rationality in Organizational Fields. American Sociological Review, 48(2), 147. https://doi.org/10.2307/2095101

Djafarova, E., \& Trofimenko, O. (2018). 'Instafamous'-credibility and self-presentation of micro-celebrities on social media. Information, Communication $\mathcal{E}$ Society, 22(10), 1432-1446. https:// doi.org/10.1080/1369118X.2018.1438491

Ellison, N., Heino, R., \& Gibbs, J. (2006). Managing Impressions Online: Self-Presentation Processes in the Online Dating Environment. Journal of Computer-Mediated Communication, 11(2), 415441. https://doi.org/10.1111/j.1083-6101.2006.00020.x

Erdogan, B.Z. (1999). Celebrity Endorsement: A Literature Review. Journal of Marketing Management, 15(4), 291-314. https://doi.org/10.1362/026725799784870379

Evans, N. J., Phua, J., Lim, J., \& Jun, H. (2017). Disclosing Instagram Influencer Advertising: The Effects of Disclosure Language on Advertising Recognition, Attitudes, and Behavioral Intent. Journal of Interactive Advertising, 17(2), 138-149. https://doi.org/10.1080/15252019.2017.1366885

Flanagin, A.J. \& Metzger, M.J. (2007). The role of site features, user attributes, and information verification behaviors on the perceived credibility of web-based information. New Media $\mathbb{E}$ Society, 9(2), 319-342. https://doi.org/10.1177/1461444807075015

Forbes (2019). The Influencer Marketing Trends That Are Coming in 2020. https://bit.ly/ForbesIMtrends

Friedman, H.H., Termini, S., \& Washington, R. (1976). The effectiveness of advertisements utilizing four types of endorsers. Journal of Advertising, 5(3), 22-24. https://doi.org/10.1080/00913367.1976.10672647

Goffman, E. (1959). The Presentation of Self in Everyday Life. Anchor.

Goldsmith, R.E., Lafferty, B.A., \& Newell, S.J. (2000). The impact of corporate credibility and celebrity credibility on consumer reaction to advertisements and brands. Journal of Advertising, 29(3), 43-54. https://doi.org/10.1080/00913367.2000.10673616

Gutiérrez, G. A., Sánchez, M. Á., \& Galiano, A. (2018). Redes sociales como medio de promoción turística en los países iberoamericanos. Retos, 15(8), 135-150. https://doi.org/10.17163/ret.n15.2018.09

Hovland, C.I., Janis, I.L., \& Kelley, H.H. (1953). Communication and persuasion; psychological studies of opinion change. Yale University Press.

Hovland, C.I., \& Weiss, W. (1951). The Influence of Source Credibility on Communication Effectiveness. Public Opinion Quarterly, 15(4), 635-650. https://doi.org/10.1086/266350

Lou, C., \& Yuan, S. (2019). Influencer Marketing: How Message Value and Credibility Affect Consumer Trust of Branded Content on Social Media. Journal of Interactive Advertising, 19(1), 58-73. https://doi.org/10.1080/15252019.2018.1533501

Miles, M. B., Huberman, M., \& Saldana, J. (2019). Qualitative Data Analysis: A Methods Sourcebook. 4th edition. Sage Publications. https://doi.org/10.1016/S0301-4622(98)00172-0

Newell, S.J., \& Goldsmith, R.E. (2001). The development of a scale to measure perceived corporate credibility. Journal of Business Research, 52(3), 235-247.

https://doi.org/10.1016/S0148-2963(99)00104-6

Nwabueze, U., \& Mileski, J. (2008). The challenge of effective governance: The case of Swiss Air. 
Corporate Governance: The International Journal of Business in Society, 8(5), 583-594. https://doi.org/10.1108/14720700810913250

OECD (2019). Country statistical profile: Chile 2019/1. https://bit.ly/statisticalprofileChile2019

Ohanian, R. (1990). Construction and validation of a scale to measure celebrity endorsers' perceived expertise, trustworthiness, and attractiveness. Journal of Advertising, 19(3), 39-52. https:// doi.org/10.1080/00913367.1990.10673191

Scott, W.R. (1995). Institutions and organizations. Sage.

Schlenker, B.R., \& Weigold, M.F. (1992). Interpersonal Processes Involving Impression Regulation and Management. Annual Review of Psychology, 43, 133-168. https://doi.org/10.1146/annurev.ps.43.020192.001025

Shiau, W.L., Dwivedi, Y.K., \& Lai, H.H. (2018). Examining the core knowledge on Facebook. International Journal of Information Management, 43, 52-63. https://doi.org/10.1016/j.ijinfomgt.2018.06.006

Silverman, D. (2000). Doing Qualitative Research: A Practical Handbook. Sage Publications,

Spiggle, S. (1994). Analysis and interpretation of qualitative data in consumer research. Journal of Consumer Research, 21(3), 491-503. https://doi.org/10.1086/209413

Stubb, C., \& Colliander, J. (2019). "This is not sponsored content" - The effects of impartiality disclosure and e-commerce landing pages on consumer responses to social media influencer posts. Computers in Human Behavior, 98, 210-222. https://doi.org/10.1016/j.chb.2019.04.024

Stubb, C., Nyström, A.G., \& Colliander, J. (2019). Influencer marketing: The impact of disclosing sponsorship compensation justification on sponsored content effectiveness. Journal of Communication Management, 23(2), 109-122. https://doi.org/10.1108/JCOM-11-2018-0119

WFA (2018). Survey on influencer marketing. https://bit.ly/surveyonIM

Wojdynski, B.W., \& Evans, N.J. (2016). Going native: Effects of disclosure position and language on the recognition and evaluation of online native advertising. Journal of Advertising, 45(2), 157168. https://doi.org/10.1080/00913367.2015.1115380

Wojdynski, B.W., Evans, N.J., \& Hoy, M.G. (2018). Measuring Sponsorship Transparency in the Age of Native Advertising. Journal of Consumer Affairs, 52(1), 115-137.

https://doi.org/10.1111/joca.12144 